\title{
ENERGY TRANSFER AND UP-CONVERSION IN THE ERBIUM ELPASOLITES $\mathrm{Cs}_{2} \mathrm{NaEr}_{x} \mathrm{Y}_{1-x} \mathrm{Cl}_{6}$
}

\author{
M. Campbell and C.D. Flint \\ Laser Laboratory, Department of Chemistry, Birkbeck College, University of London \\ 29 Gordon Square, London, WC1H OPP, U.K.
}

The luminescence decay curves from the ${ }^{4} F_{9 / 2}$ and ${ }^{4} I_{9 / 2}$ states of the cubic hexachloroelpasolite crystals $\mathrm{Cs}_{2} \mathrm{NaEr}_{x} \mathrm{Y}_{1-x} \mathrm{Cl}_{6}(x=0.001 \div 1)$ have been measured over the temperature range $10-300 \mathrm{~K}$. The ${ }^{4} I_{9 / 2}$ state undergocs an electric dipole vibronic-electric dipole vibronic cross-relaxation process at $300 \mathrm{~K}$, but this mechanism is inefficient below $165 \mathrm{~K}$. Excitation into the ${ }^{4} F_{9 / 2}$ state, results in emission in the green, blue and ultra violet regions. The up-conversion processes which could account for the ultraviolet emission are discussed.

PACS numbers: 13.40.Hq, 31.70.Hq, 34.30.th, 61.72.Ss, 78.55.Hx

\section{Introduction}

The cubic erbium hexachloroelpasolite is a useful system for the study of energy transfer processes for a number of reasons. Firstly, the erbium ions occupy sites of exact octahedral symmetry and consequently pure electric dipole radiative $f-f$ transitions are forbidden. Overall $f-f$ transition probabilities (from magnetic dipole and vibronic transitions) are relatively small.

This leads to long lived excited states and therefore facilitates the observation of energy transfer processes. Secondly, in the elpasolite lattice, the phonon cut-off frequency is smaller than $300 \mathrm{~cm}^{-1}$. This reduces the non-radiative transition rates between levels and therefore luminescence is observed from many excited states. Thirdly, the octahedral crystal field means that the number of crystal field levels is minimized. This lowers the number of possible interaction pathways leading to energy transfer processes and allows the mechanisms to be determined more precisely.

The energy levels of erbium both, as a free ion and in the elpasolite lattice, have been extensively investigated and are well catalogued $[1,2]$. The odd parity vibrational modes of the $\mathrm{ErCl}_{6}^{3+}$ moiety, $\nu_{6}, \nu_{4}$ and $\nu_{3}$, occur at about 80, 120, and $280 \mathrm{~cm}^{-1}$ and are readily observed in the vibronic transitions. There are a large number of higher energy levels of $\mathrm{Er}^{3+}$ in the visible and near infrared regions (Fig. 1), many having similar energy gaps between them. This ladder like structure, coupled with the fact that these states can be extremely long lived 


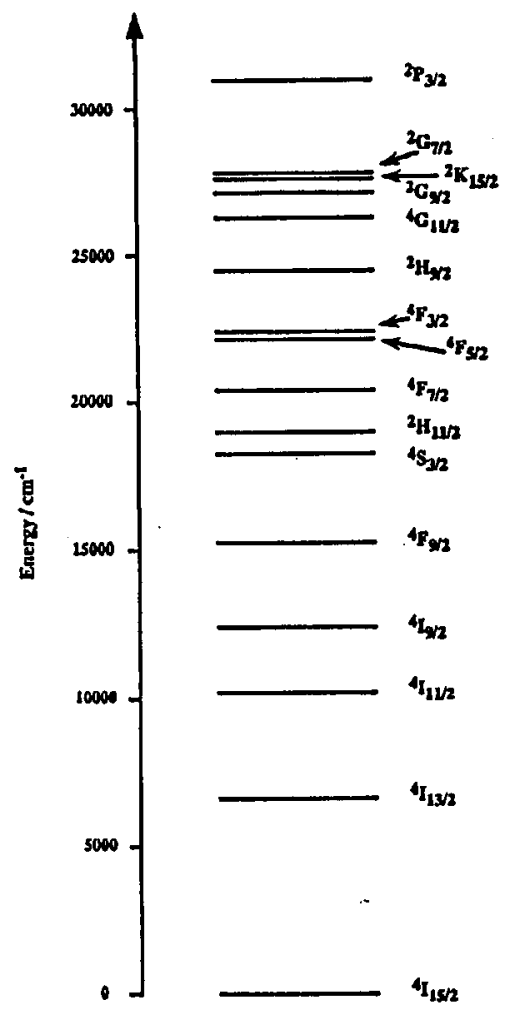

Fig. 1. Energy levels of $\mathrm{Er}^{3+}$.

means that there are a large number of energy transfer possibilities which may lead to cross relaxation and up-conversion processes.

In this paper we present the decay kinetics of the ${ }^{4} F_{9 / 2}$ and ${ }^{4} I_{9 / 2}$ states of this system, and the up-conversion processes resulting from the population of these states which have not been reported previously $[3,4]$.

\section{Experimental}

Luminescence decay curves were measured from single crystals of $\mathrm{Cs}_{2} \mathrm{NaEr}_{x} \mathrm{Y}_{1-x} \mathrm{Cl}_{6}$, synthesized using a modified Bridgman method, as described previously [5]. Excitation was into the ${ }^{4} F_{9 / 2}$ state at $15360 \mathrm{~cm}^{-1}$ and decay curves were measured over the temperature range 10-300 K and for the concentration range $x=0.001 \div 1.0$. The emission from the ${ }^{4} I_{9 / 2}$ state is conspicuously long lived (lifetime $>0.1 \mathrm{~s}$ at low temperatures in dilute samples) and it is necessary therefore to use very low laser pulse repetition rates $\left(<2 \mathrm{~s}^{-1}\right)$ if excited state absorption of a second laser pulse is to be avoided. 


\section{Decay of excited ${ }^{4} F_{9 / 2}$ states}

The luminescence decay curves of the ${ }^{4} F_{9 / 2}$ state were measured at $14950 \mathrm{~cm}^{-1}$ (the ${ }^{4} F_{9 / 2} \rightarrow{ }^{4} I_{15 / 2}$ transition) and were exponential for all concentrations and temperatures. At $300 \mathrm{~K}$ the decay constants are almost independent of concentration (Fig. 2). At lower temperatures, the decay constant decreases and becomes dependent on the concentration. At low temperatures there are no two-ion, two phonon cross-relaxation processes available, so cross relaxation will be inefficient. At the lowest concentrations, when the erbium ions are effectively separated from one another, the change in the decay constant with temperature (Fig. 3) is consistent with the coth law for vibronic transitions, although the thermal population of different components of the ${ }^{4} F_{9 / 2}$ state will also contribute to this temperature dependence. The concentration dependence occurs rather abruptly between $x=0.15$ and $x=0.25$, in this concentration range almost every erbium ion has at least one other erbium ion as a nearest neighbour and it is likely that energy migration amongst the donor ions is responsible for the change in behaviour. This would be consistent with the exponential decay curves at intermediate values of $x$.
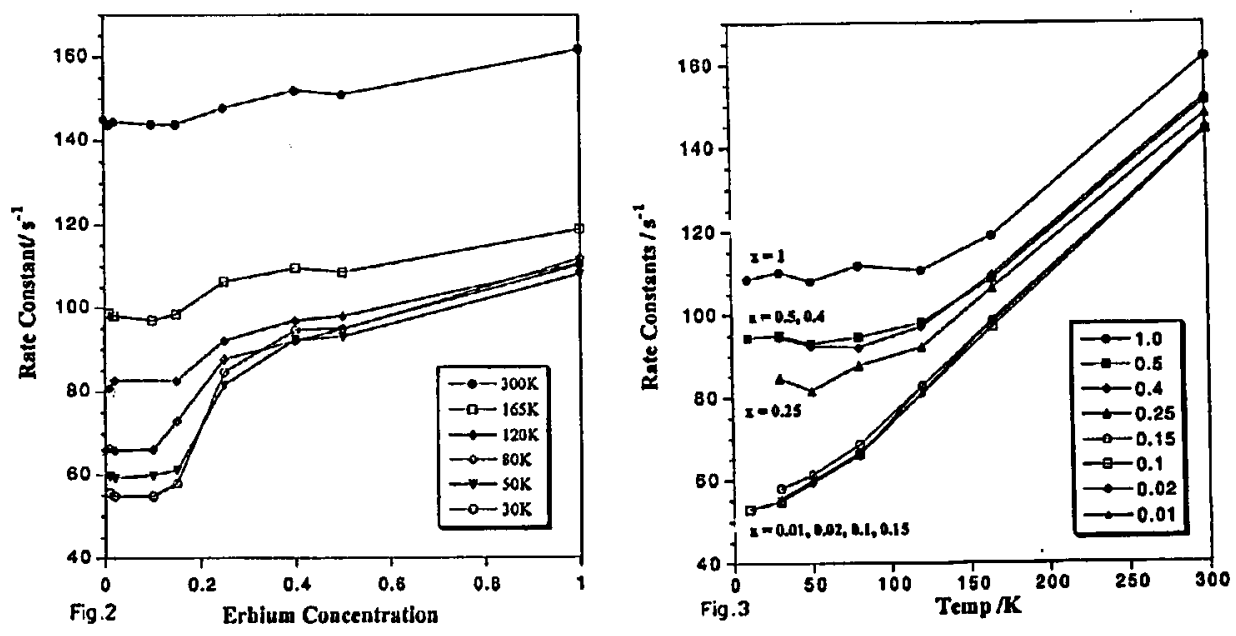

Fig. 2. The concentration dependence (erbium mole fraction) of the decay constants of the excited ${ }^{4} F_{9 / 2}$ state (measured at $14950 \mathrm{~cm}^{-1}$ ) for $\mathrm{Cs}_{2} \mathrm{NaEr}_{x} \mathrm{Y}_{1-x} \mathrm{Cl}_{6}$ at various temperatures.

Fig. 3. The temperature dependence of the decay constants of the excited ${ }^{4} F_{9 / 2}$ state of $\mathrm{Cs}_{2} \mathrm{NaEr}_{x} \mathrm{Y}_{1-x} \mathrm{Cl}_{6}$ (measured at $14950 \mathrm{~cm}^{-1}$ ) for various mole fractions of erbium.

\section{Decay of the excited ${ }^{4} I_{9 / 2}$ states}

The ${ }^{4} I_{9 / 2}$ state of erbium is populated by decay from the ${ }^{4} F_{9 / 2}$ state, the energy gap between these two levels being approximately $2650 \mathrm{~cm}^{-1}$ which corresponds to about 9 of the highest energy vibrations in this lattice. The non-radiative transition between the two states is therefore expected to be relatively unimportant and the ${ }^{4} I_{9 / 2}$ can be assumed to be populated predominantly by the radiative 
transition from the ${ }^{4} F_{9 / 2}$ state. As expected the luminescence decay curves (measured at $12240 \mathrm{~cm}^{-1}$ ) show a slow rise with a constant equal to the decay constant of the ${ }^{4} F_{9 / 2}$ state followed by a conspicuously slow exponential decay.

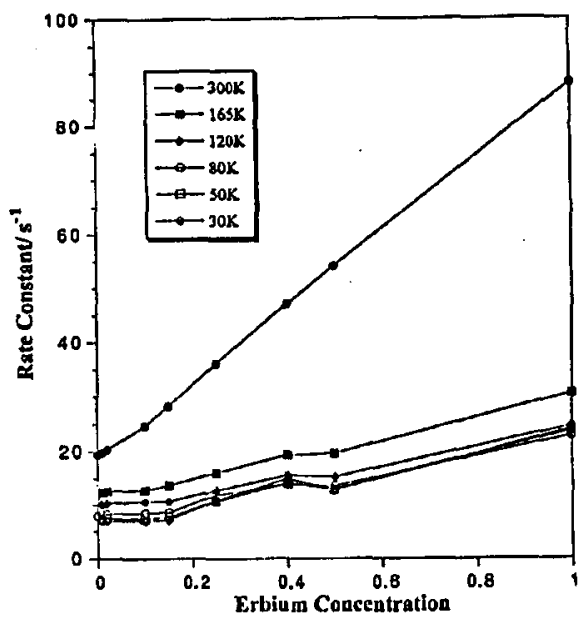

Fig. 4. The concentration dependence (erbium mole fraction) of the decay constants of the excited ${ }^{4} I_{9 / 2}$ state (measured at $12240 \mathrm{~cm}^{-1}$ ) of $\mathrm{Cs}_{2} \mathrm{NaEr}_{x} \mathrm{Y}_{1-x} \mathrm{Cl}_{6}$ for different temperatures.

At low temperatures $(<165 \mathrm{~K})$ there is only a slight concentration quenching occurring (Fig. 4). At higher temperatures there is an almost linear increase in the decay rate constant with concentration. This is attributed to the cross-relaxation mechanism

$$
{ }^{4} I_{9 / 2}+{ }^{4} I_{15 / 2}+\nu_{\mathrm{u}} \rightarrow{ }^{4} I_{13 / 2}+{ }^{4} I_{13 / 2}+\nu_{\mathrm{u}}^{\prime} .
$$

This mechanism requires the thermal population of higher crystal field levels as well as the absorption of a phonon on either or both the donor and acceptor levels. A distribution over these levels is consistent with the observed temperature dependence.

The shell model describing the decay of the donor emission in the presence of acceptors may be written [6]

$$
I(t)=I(0) \exp \left(-k_{0} t\right) \prod_{r_{n}}^{\text {all shells }} \sum_{r_{n}}^{N_{n}} O_{r_{n}}^{N_{n}}(x) \exp \left[-r_{n}\left(\frac{R_{1}}{R_{n}}\right)^{6} k^{\mathrm{ET}} t\right],
$$

where $x$ is the mole fraction of acceptors, $k_{0}$ is the intrinsic decay of the isolated ion, $N_{n}$ is the maximum number of ions in the $n$-th shell, $r_{n}$ is the occupancy factor, $O_{r_{n}}^{N_{n}}(x)$ is the probability of a particular distribution of acceptors, $R_{1} / R_{n}$ the ratio of the distance of the nearest neighbour shell to the $n$-th shell, and $k^{\text {ET }}$ is the energy transfer rate to a single ion at the nearest neighbour position. It can be shown [6] that for the $x=1$ case (i.e. $100 \%$ erbium) the decay is exponential with a decay of $k_{0}+14.42 k^{\mathrm{ET}}$. Using this relationship (which also applies in the 
presence of migration amongst the donors) we find that $k^{\mathrm{ET}}=4.6 \mathrm{~s}^{-1}$ at $300 \mathrm{~K}$. Although this is a very slow rate of energy transfer, it is observable due to the extremely long lifetime of the ${ }^{4} I_{9 / 2}$ state which is $51.5 \mathrm{~ms}\left(k=19.4 \mathrm{~s}^{-1}\right)$ for the isolated ion at $300 \mathrm{~K}$ and $151.5 \mathrm{~ms}$ at $10 \mathrm{~K}\left(k=6.6 \mathrm{~s}^{-1}\right)$.

\section{Up-conversion processes}

Under red excitation, emission was also observed at shorter wavelength than the exciting wavelength, with luminescence occurring in the ultraviolet, blue and green regions at $25975,24100,22075,20410$ and $17860 \mathrm{~cm}^{-1}$. The emission at $25975 \mathrm{~cm}^{-1}$ which is the subject of this paper was approximately 4 or 5 times more intense than that at $24100 \mathrm{~cm}^{-1}$ and at least two orders of magnitude greater than the other emissions. The time evolution of the lower states populated by up-conversion is more complex and will be discussed elsewhere.

A study of the power dependence of the emission intensity at $25975 \mathrm{~cm}^{-1}$ gives a quadratic dependence upon the input laser power implying a two-photon mechanism for this red to ultraviolet up-conversion. The only possible excited state for this emission is then the ${ }^{4} G_{11 / 2}$ and the up-conversion must involve the ${ }^{4} F_{9 / 2}$ state. For $x=1$ the luminescence decay curve shows a slow rise $(5 \mathrm{~ms}$ at $80 \mathrm{~K})$ at all temperatures, but for $x=0.1$ a much faster (but clearly observable, $200 \mu \mathrm{s}$ at $80 \mathrm{~K}$ ) rise occurs at all temperatures. At intermediate concentrations there is a monotonic transition between these two types of behaviour. The contributions of these two processes are temperature dependent (Figs. 5, 6, 7).

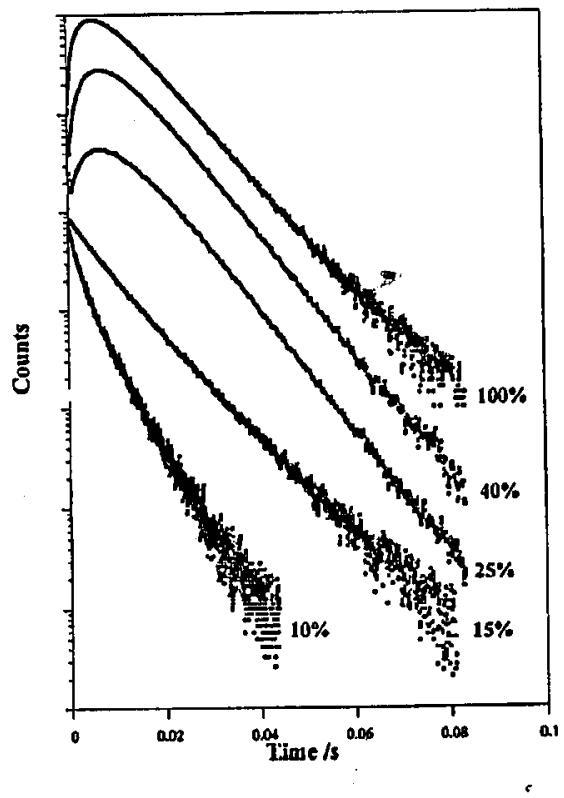

Fig. 5. $80 \mathrm{~K}$ ultraviolet luminescence decay curves (measured at $25974 \mathrm{~cm}^{-1}$ ) with excitation at $15360 \mathrm{~cm}^{-1}$ for various mole fractions of erbium in $\mathrm{Cs}_{2} \mathrm{NaEr}_{x} \mathrm{Y}_{1-x} \mathrm{Cl}_{6}$. 


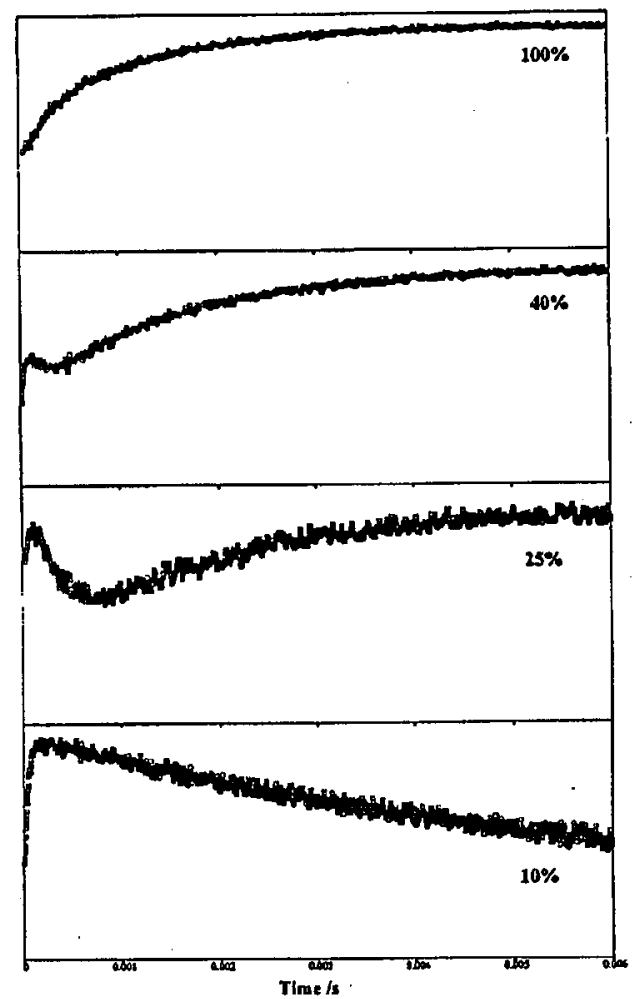

Fig. 6. Initial components of the $80 \mathrm{~K}$ ultraviolet luminescence decay curves (measured at $25974 \mathrm{~cm}^{-1}$ ) with excitation at $15360 \mathrm{~cm}^{-1}$ for various mole fractions of erbium in $\mathrm{Cs}_{2} \mathrm{NaEr}_{0.1} \mathrm{Y}_{0.9} \mathrm{Cl}_{6}$.

Processes which might contribute to emission from the ${ }^{4} G_{11 / 2}$ state include: (1) Energy transfer from one excited ion to another already excited ion ${ }^{4} F_{9 / 2}+{ }^{4} I_{9 / 2} \rightarrow{ }^{4} I_{15 / 2}+{ }^{2} K_{15 / 2}$

followed by fast relaxation from the ${ }^{2} K_{15 / 2}$ level to the ${ }^{4} G_{11 / 2}$.

(2) Energy transfer from one excited ion to another already excited ion ${ }^{4} F_{9 / 2}+{ }^{4} F_{9 / 2} \rightarrow{ }^{4} I_{15 / 2}+{ }^{2} G_{7 / 2}$

followed by fast relaxation from the ${ }^{2} G_{7 / 2}$ level to the ${ }^{4} G_{11 / 2}$.

(3) Excited state absorption from the ${ }^{4} F_{9 / 2}$ state excited during the same laser pulse leading to population of the ${ }^{2} G_{7 / 2}$ level followed by fast relaxation from the ${ }^{2} G_{7 / 2}$ level to the ${ }^{4} G_{11 / 2}$.

If we assume fast migration amongst the donors and restrict energy transfer to nearest neighbour interactions (within the shell model $83 \%$ of the energy transfer process occur between nearest neighbours for dipole- dipole interactions) then the rate equations describing the populations of these levels for Process (1) are

$$
\frac{\mathrm{d} n_{3}}{\mathrm{~d} t}=X n_{1}-k_{3} n_{3}-W n_{3} n_{2}
$$



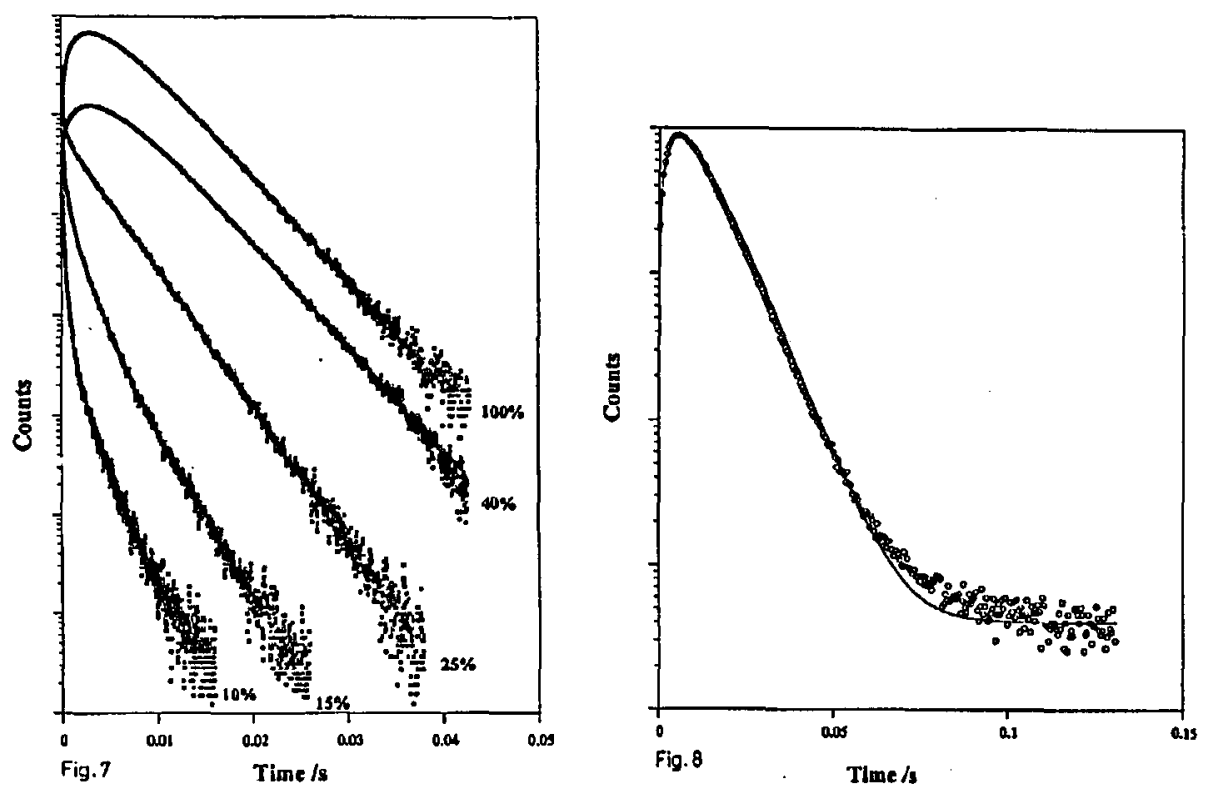

Fig. 7. $300 \mathrm{~K} \mathrm{UV} \mathrm{luminescence} \mathrm{decay} \mathrm{curves} \mathrm{(measured} \mathrm{at} 25974 \mathrm{~cm}^{-1}$ ) with excitation at $15360 \mathrm{~cm}^{-1}$ for various mole fractions of erbium in $\mathrm{Cs}_{2} \mathrm{NaEr}_{0.1} \mathrm{Y}_{0.9} \mathrm{Cl}_{6}$.

Fig. $880 \mathrm{~K}$ ultraviolet luminescence decay curves (measured at $25974 \mathrm{~cm}^{-1}$ ) with excitation at $15360 \mathrm{~cm}^{-1}$ for $\mathrm{Cs}_{2} \mathrm{NaErCl}_{6}$. The solid line was calculated a priori using Eq. (6).

$$
\begin{aligned}
& \frac{\mathrm{d} n_{2}}{\mathrm{~d} t}=\zeta k_{3} n_{3}-k_{2} n_{2}-W n_{3} n_{2}, \\
& \frac{\mathrm{d} n_{4}}{\mathrm{~d} t}=2 W n_{3} n_{2}-k_{4} n_{4},
\end{aligned}
$$

where the levels ${ }^{4} I_{15 / 2},{ }^{4} I_{9 / 2},{ }^{4} F_{9 / 2}$ and ${ }^{4} G_{11 / 2}$ are labelled $1,2,3$ and 4 , respectively and $k_{i}$ is the intrinsic decay constant for the $i$-th level and $n_{i}$ is the population of the $i$-th level. $X$ is the rate of excitation of the erbium ions and is the product of $\mathrm{Er}^{3+}$ absorption cross section and the incident photon flux and $W$ is the rate of energy transfer leading to the up-conversion. $\zeta$ is the branching ratio for the transition to the ${ }^{4} I_{9 / 2}$ state.

The acceptor is excited to the ${ }^{2} K_{15 / 2}$ state from which rapid thermal relaxation occurs to the ${ }^{4} G_{11 / 2}$ state which is responsible for the luminescence at about $25975 \mathrm{~cm}^{-1}$. Therefore, in the preceding rate equations, the terms for back transfer have been neglected since they will be extremely small. The re-population of the ${ }^{4} F_{9 / 2}$ and ${ }^{4} I_{9 / 2}$ states from the higher energy ${ }^{4} G_{11 / 2}$ state has been neglected.

To obtain an approximate analytical solution to these coupled nonlinear differential equations, the last terms in Eqs. (1) and (2) are assumed to be relatively small and are neglected. For a $\delta$ function pulse at time zero the approximate solutions are

$$
n_{3}(t)=n_{3}(0) \exp \left(-k_{3} t\right),
$$




$$
\begin{aligned}
n_{2}(t) & =\frac{n_{3}(0) \zeta k_{3}}{k_{2}-k_{3}}\left[\exp \left(-k_{3} t\right)-\exp \left(-k_{2} t\right)\right], \\
n_{4}(t) & =\frac{2 n_{3}(0)^{2} W \zeta k_{3}}{k_{2}-k_{3}} \\
\times & {\left[\frac{\exp \left(-2 k_{3} t\right)-\exp \left(-k_{4} t\right)}{k_{4}-2 k_{3}}+\frac{\exp \left(-k_{4} t\right)-\exp \left(-k_{3}-k_{2}\right) t}{k_{4}-k_{3}-k_{2}}\right] . }
\end{aligned}
$$

Similarly, it is possible for the up-conversion to occur via Process (2)

$$
\begin{aligned}
\frac{\mathrm{d} n_{3}}{\mathrm{~d} t} & =X n_{1}-k_{3} n_{3}-W n_{3} n_{3}, \\
\frac{\mathrm{d} n_{4}}{\mathrm{~d} t} & =W n_{3} n_{3}-k_{4} n_{4} .
\end{aligned}
$$

With the assumptions as above the approximate solutions to these rate equations are

$$
\begin{aligned}
& n_{3}(t)=n_{3}(0) \exp \left(-k_{3} t\right) \\
& n_{4}(t)=\frac{n(0)^{2} W}{k_{4}-2 k_{3}}\left[\exp \left(-2 k_{3} t\right)-\exp \left(-k_{4} t\right)\right] .
\end{aligned}
$$

For Process (3) the excited state absorption occurs within the time of the laser pulse which is smaller than 7 ns and therefore no measurable rise time is expected for the build-up in the population of the ${ }^{2} G_{7 / 2}$ state. Excited state absorption within the same laser pulse is not expected from any state lower than ${ }^{4} F_{9 / 2}$ state since there is no significant population of these states within the $7 \mathrm{~ns}$ laser pulse. Note that the repetition rate of the laser was slow enough so that the long lived excited states were not populated from a second laser pulse.

The decay curves of the emission from the ${ }^{4} G_{11 / 2}$ as a function of concentration show a sharp change in mechanism of the up-conversion process with concentration (Figs. 5,6,7). This change occurs at low temperatures between $x=0.15$ and 0.25 . At higher temperatures this change in behaviour occurs at higher concentrations.

To distinguish between these processes, we have attempted to model the predicted time evolution of the emission and compare it with the experimental results. Figure 8 shows the time evolution of the emission from the ${ }^{4} G_{11 / 2}$ state for the $x=1$ crystal at $80 \mathrm{~K}$, compared with the calculated decay curve for Process (1). The agreement is excellent considering the approximations involved and that there is no curve fitting, the rates $k_{2}$ and $k_{3}$ having been directly measured previously. Provided that $k_{4}$ is large enough that the denominators in the bracketed terms in Eq. (6) have the same sign then the decay curves are almost independent of $\boldsymbol{k}_{4}$. The agreement between the a priori calculated curves and the experimental data strongly suggests that Process (1) is dominant in this concentration and temperature regime. Models based on Process (2) fail to reproduce the experimental data in this regime.

For $x=0.1$ the decay curves and hence the mechanism is clearly different. There is still a finite rise time which eliminates excited state absorption as a 


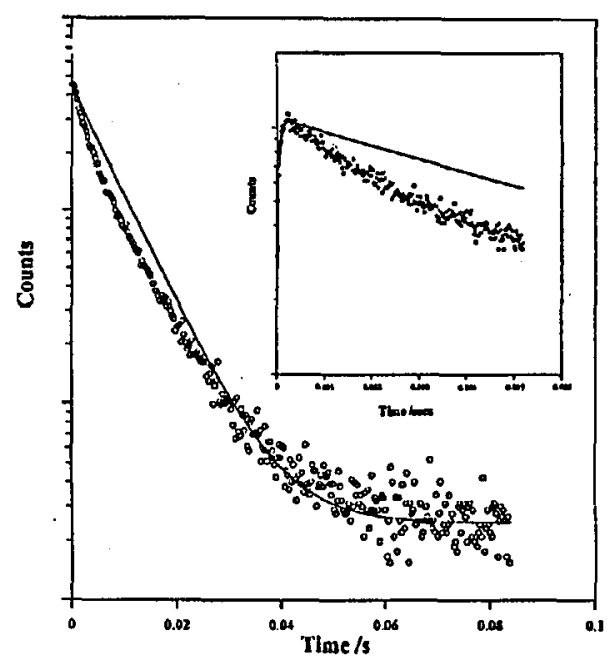

Fig. 9. $80 \mathrm{~K}$ ultraviolet luminescence decay curves (measured at $25974 \mathrm{~cm}^{-1}$ ) with excitation at $15360 \mathrm{~cm}^{-1}$ for $\mathrm{Cs}_{2} \mathrm{NaEr}_{0.1} \mathrm{Y}_{0.9} \mathrm{Cl}_{6}$. The solid line was calculated a priori using Eq. (10). The inset shows the short time behaviour.

possible mechanism. The calculated curves based on Process (1) fail to predict the time evolution of the emission for this concentration. However a priori calculated decay curves based on Process (2) (using the separately measured values of $k_{3}$ ) have a general resemblance to the measured data but the decay following the initial rise is faster than predicted (Fig. 9). A possible explanation is the neglect of the third term in Eq. (7) which will lead to enhanced depopulation of state 3 when $n_{3}$ is relatively large, i.e. during the early part of the decay curve.

\section{Conclusions}

Excitation into the ${ }^{4} F_{9 / 2}$ state of $\mathrm{Er}^{3+}$ in $\mathrm{Cs}_{2} \mathrm{NaEr}_{x} \mathrm{Y}_{1-x} \mathrm{Cl}_{6}(x=0.001 \div 1)$ results in strong emission from this level with little cross relaxation even for $x=1$. This state decays to the remarkably long lived, ${ }^{4} I_{9 / 2}$ state which has an emissive lifetime of $0.125 \mathrm{~s}$. Up-conversion processes involving both these states lead to emission in the ultraviolet region. The kinetics of the decay curves from the state populated by up-conversion shows that for $x=1$ both the ${ }^{4} F_{9 / 2}$ and ${ }^{4} I_{9 / 2}$ states are involved whereas at low concentrations only the ${ }^{4} F_{9 / 2}$ state is responsible.

We thank the EPSRC for the award of a postgraduate grant to MC.

\section{References}

[1] Z. Hasan, F.S. Richardson, Molec. Phys. 45, 1299 (1982).

[2] P.A. Tanner, Molec. Phys. 63, 365 (1988).

[3] W. Ryba-Romanowski, Z. Mazurak, B. Jeżowska-Trzebiatowska, J. Lumin. 27, 177 (1982).

[4] W. Ryba-Romanowski, G. Dominiak-Dzik, S. Golab, J. Phys., Condens. Matter 6, 1593 (1992). 
[5] M. Bettinelli, C.D. Flint, J. Phys., Condens. Matter 2, 8417 (1990).

[6] S.O. Vasquez, C.D. Flint, Chem. Phys. Lett. 238, 378 (1995). 